\title{
Expression and Sequence Analysis of HTH in Blackberry (Rubus Spp.)
}

\author{
Xunju Liu ${ }^{1}$, Min Zhou ${ }^{1}$, Yan Wang ${ }^{2}$, Haoru Tang ${ }^{2}$ and Qing Chen ${ }^{1, \text { a, }}$ * \\ ${ }^{1}$ College of Horticulture, Sichuan Agricultural University, Sichuan, China; \\ 2 Institute of Pomology and Olericulture, Sichuan Agricultural University, Sichuan, China \\ a supnovel@gmail.com
}

Keywords: Rubus spp, HTH, Cloning and expression, Light irradiation.

\begin{abstract}
To better understand the molecular mechanisms of cuticle wax formation in blackberry, the waxy synthetic related gene $H T H$ was cloned from blackberry. The open reading frame of this gene was $1785 \mathrm{bp}$, encoding 594 amino acid residues with a molecular weight of $64.6 \mathrm{kD}$ and a theoretical isoelectric point of 9.39. Its amino acid sequence has the highest identity with strawberry. The results of real-time quantitative PCR showed that the expression level of HTH gene decrease rapidly with the maturity of the fruit. The expression level of $H T H$ decreased with the increase of red or blue light intensity. The lowest expression of $\mathrm{HTH}$ gene was under pure blue light treatment.
\end{abstract}

\section{Introduction}

Cuticle is a hydrophobic protective layer coated directly on the surface of terrestrial plants in contact with the air. The components of the cuticle waxy complex, mainly linear aliphatic, its derivatives, terpenoids and flavonoids secondary metabolites [1]. Plant epidermis wax plays an important role in plant adaptation to biotic stresses and abiotic stresses. It limits plant non-stomatal transpiration and enhances plant drought resistance. It helps plants to clean themselves, reducing the attachment of dust, pollen and spores, preventing plants from being exposed to excessive UV radiation, affecting plant-pathogen and plant-insect interactions, ensuring plant organs Normal demarcation thus prevents plant organ fusion [2].

The HOTHEAD (HTH) gene encodes a flavonoid adenine dinucleotide oxidoreductase. Krolikowski KA found HTH gene limits the interaction between epidermal cells in 2003[3]. After that Kurdyukov S found HTH gene ensures the integrity of the outer epidermal cell wall by regulating the waxy synthesis of Arabidopsis [4]. Xu Y found the HTH gene regulates rice pollen development by limiting the fusion of epidermal cells [5]. The HTH gene, like other waxy genes, is located in the endoplasmic reticulum [6]. The expression of HTH gene in different tissues of rice was analyzed. The results showed that HTH gene was expressed in all tissues of rice, but the expression was highest in young shoots [7]. The function of HTH gene, the mechanism of action of HTH gene and its regulatory mechanism are not clear yet, more in-depth testing is needed.

Many of the wild resources of Rubus plant fruit surface and the stems are covered with a thick waxy layer, which is highly resistant to sun light. However, there are very few cultivars of raspberry cultivars that have such characteristics. Therefore, preventing fruit from sunburn is still one of the measures that must be taken in the fruit production. In this study, the spineless blackberry 'Arapaho' was used as a material. From the transcriptomic data from previous research in our group, the transcript sequence homologous to Arabidopsis HTH gene was extracted. Gene-specific primers were designed and the HTH gene was cloned by RT-PCR. The sequence of HTH gene in blackberry was analyzed by bioinformatics method. The expression of HTH gene in blackberry was analyzed by qRT-PCR. The expression of HTH gene in blackberry leaves under different light conditions was analyzed by qRT-PCR. Prediction of HTH gene in the formation of blackberry waxy important role in revealing plant epidermal waxy and environmental interaction mechanism and breeding varieties provide theoretical basis for resistance. 


\section{Materials and Methods}

\subsection{Materials}

In April of 2015, the young leaves and fruits of blackberry cultivar 'Arapaho', which are planted at the teaching and scientific research base of Sichuan Agricultural University were taken as materials and frozen in liquid nitrogen for RNA extraction. Fruits of three maturity level were included: young fruits ( 5 days post anthesis, DPA), red fruits (20 DPA), and black fruits (25 DPA). Fruits of the same developmental stage were randomly selected on different branches of the same shrub. 20 fruits were randomly selected as one biological replicate. Three replicates were conducted for each stage.

The 'Arapaho' sterile seedlings were established from April 2015. Plants were subcultured every four weeks in MS medium supplemented with $20 \mathrm{~g} / \mathrm{L}$ sucrose. Plants were cultured in a standard tissue culture room with temperature $25^{\circ} \mathrm{C} \pm 2{ }^{\circ} \mathrm{C}, 16 \mathrm{~h}$ light/day, $70 \% \mathrm{RH}$.

\subsection{HTH Gene Cloning and Sequencing}

The young leaves, three maturity fruits were powdered in liquid nitrogen. Total RNA was extracted by modified CTAB method [8]. cDNA was synthesized according to the instructions of the Reverse Script kit Prime Script RT reagent Kit with gDNA Eraser. From the transcriptome data of our previous research (Bioproject PRJNA401210), a transcript homologous to Arabidopsis thaliana HTH gene was extracted. Two specific primers were designed in Primer 5.0 software (HTH-F: GCGTTGATAATGGCTTCGGTTAAT and HTH-R;CTTCCTTTTATTATATACCAGCTGCTCT

TCC, the underline is the start codon).

PCR amplification was conducted in $20 \mu \mathrm{L}$ reaction mixture. PCR products were detected by $1 \%$ agarose gel electrophoresis. After the recovering from the gel, the specific PCR amplicon was ligated into pEASY-Blunt vector and transformed into E. coli Trans 1-T1. After overnight culture and single colonies were picked for detection. The positive clones were sent to Sangon Biotech for sequencing.

\subsection{Bioinformatic Analysis}

The open reading frame was searched with NCBI ORF finder. Protein molecular weight and theoretical isoelectric point were analyzed with ProtParam. Conserved domains were screened by NCBI-CDD. Multiple sequence alignments were performed with MAFT. Phylogenetic tree was constructed by IQTREE.

\subsection{Real-time Quantitative Analysis of HTH Gene Expression}

The blackberry actin gene was used as an internal reference (Qactin-F: AGACTACATACAACT CCATCAT and Qactin-R: ACCAATCCAGACACTATACTT). The RT-PCR primer was designed based on the sequenced HTH gene sequence, Qcer-F: TGCCAATTCCAATGTCTCA and Qhth-R: AACCTTGTGCTTGCTCTA.

The expression of $H T H$ gene in different fruit maturity level and under different light irradiation conditions. Thirty-day old subculture seedlings of 'Arapohol' were placed under top red light (660 $\mathrm{nm}$ ), blue light (470 nm), combinations of different quantity of the two (R-B 2:8, R-B 5:5, R-B 8:2) in a growth chamber which was equipped with LED. Light intensity was set to $50001 \mathrm{x}, 25{ }^{\circ} \mathrm{C}, 16 \mathrm{~h}$ light/day. Plants grow under white light were used as controls. Leaves at the same node level were collected after $72 \mathrm{~h}$ of irradiation. Three replicates were set up for each treatment. Total RNA was extracted for analysis of the expression of $H T H$ gene under such conditions.

RT-qPCR reaction mixture consists of SYBR Green fluorescent dye $10 \mu \mathrm{L}$, the upstream and downstream primers $0.8 \mu \mathrm{L}$, cDNA $1.5 \mu \mathrm{L}$, $\mathrm{ddH}_{2} \mathrm{O}$ to $20 \mu \mathrm{L}$. Reaction were conducted at $95{ }^{\circ} \mathrm{C}$ for 3 min, followed by 40 PCR cycles of $95{ }^{\circ} \mathrm{C} 10 \mathrm{~s}, 55^{\circ} \mathrm{C} 30 \mathrm{~s}, 72{ }^{\circ} \mathrm{C} 15 \mathrm{~s}$. Fluorescence was collected at the third step of each cycle. Reactions with no template cDNA were used as negative controls. All PCR reactions were replicated three times, and the data were analyzed by $2^{-\Delta \Delta \mathrm{Ct}}$ method. The relative expression values were subjected to One-way ANOVA analysis in SPSS software (v20.0). 


\section{Results}

\subsection{HTH Gene in Rubus Spp.}

The $H T H$ gene homologous to Arabidopsis $H T H$ gene was successfully identified in Rubus spp. The open reading frame of this gene was $1785 \mathrm{bp}$, encoding a peptide of 594 amino acid residues with a molecular weight of $64.6 \mathrm{kD}$. Theoretical isoelectric point is 9.39. Multiple sequence alignment was conducted with the currently identified other HTH proteins (Fig. 1).

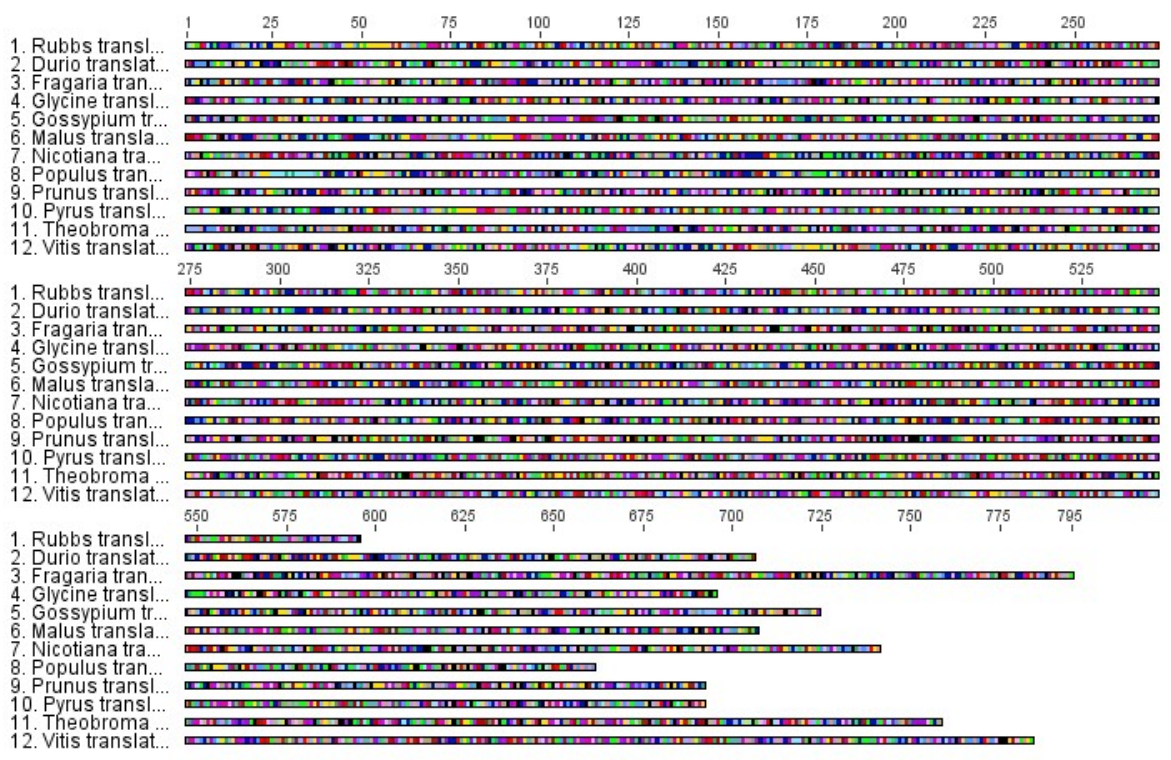

Fig. 1 Sequence alignment of HTH related proteins.

\subsection{Phylogeny of HTH Protein}

The phylogeny of HTH related proteins from species like Malus, Pyrus, and Citrus were referred by most likelihood (ML) trees (Fig. 2). The blackberry HTH protein has the highest similarity with the amino acid sequence of strawberry HTH protein, indicating the closest evolutionary relationship with strawberry. Rosaceae species formed a unique clade, but can be grouped into two clusters with these species in this investigation.

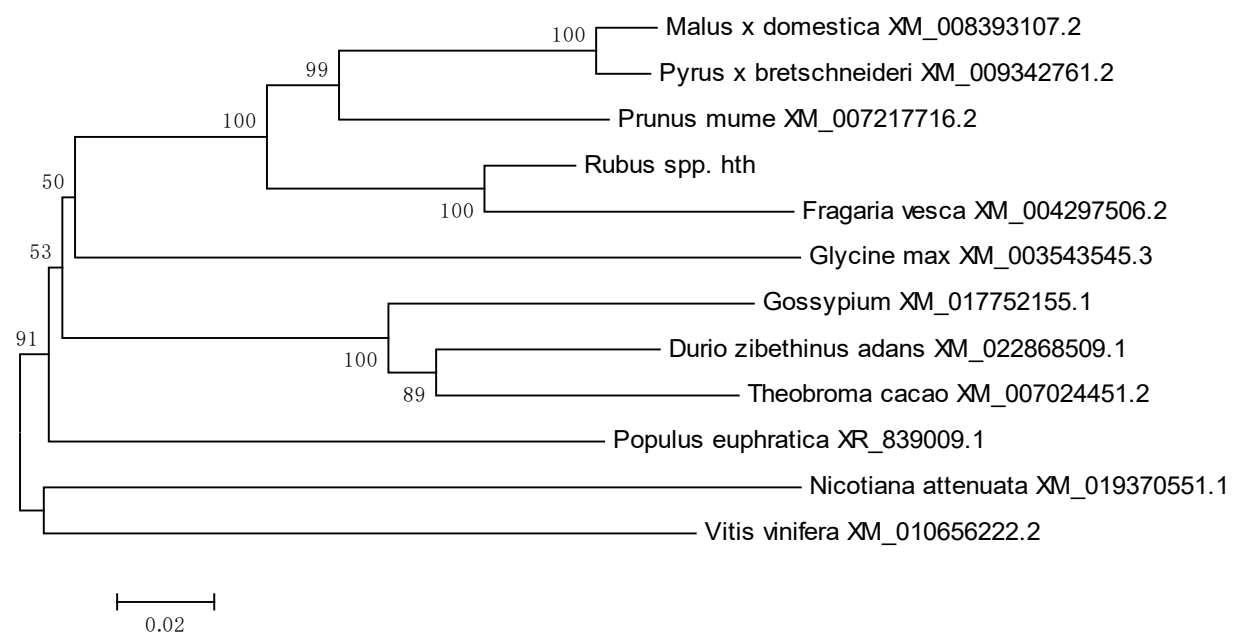

Fig. 2 ML tree of HTH of blackberry and other plants.

\subsection{HTH Gene Expression in Different Developmental Stages of Fruit}

The RT-qPCR was used to access the expression of HTH gene during blackberry fruit development. As is demonstration in Fig. 3A, the expression level of $H T H$ gene decreased rapidly with the maturity of the fruit. This shows that the $H T H$ gene may be involved in the regulation of fruit development in the early formation of related substances. 


\subsection{Light-induced HTH Expression}

The blackberry seedlings were treated with six different light conditions respectively. The expression of $H T H$ gene was analyzed by qRT-PCR (Fig. 3B). After treatment with red and blue light, the expression of HTH gene was decreased. There was no significant difference between the treatment of R-B 5:5 and the control, but under the four treatments of full red light, full blue light, R-B 8:2 and R-B 2:8, Compared with the control (all white light), the expression levels of $H T H$ gene were significantly different. This may indicate that red and blue light inhibit the expression of $H T H$ gene in blackberry.
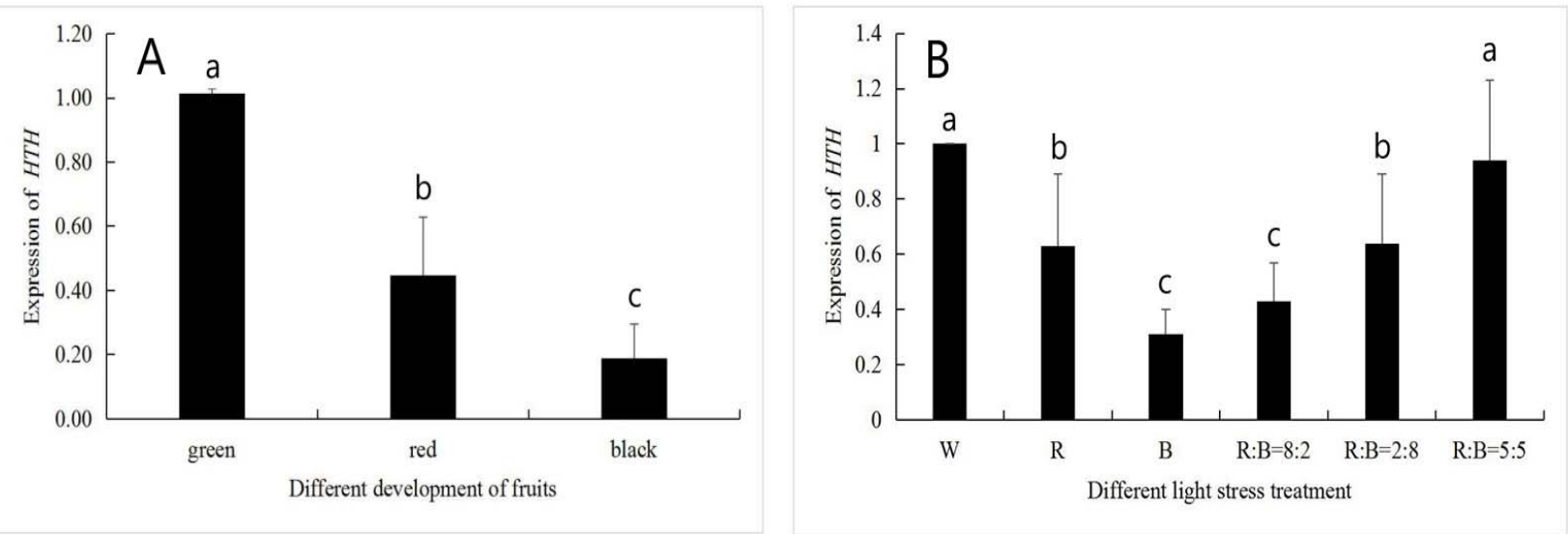

Fig. 3 Expression profiling of HTH gene in different fruit development stages and responses to different light irradiation in blackberry leaves. Same letters indicate non-significant differences among stages (One-way ANOVA, $\mathrm{p}<0.05$ ).

\section{Conclusion}

The BlackBerry HTH protein sequence obtained in this study has high similarity with the HTH protein sequences of other species and has the closest evolutionary relationship with strawberry. Using qRT-PCR to determine the expression level of $H T H$ gene in different developmental stages of fruit, the results showed that the expression level of $H T H$ gene gradually decreased along with the fruit ripening, which was consistent with the results of Comai L [9].In this study, blackberry seedlings were treated with six different light combinations. The results showed that the expression of $H T H$ gene in blackberry leaves treated with red and blue light decreased significantly, indicating that illumination affected the expression of $\mathrm{HTH}$ gene, but the specific regulation Mechanism, need further study.

\section{References}

[1]. Fich E A, Segerson N A, Rose J K. The Plant Polyester Cutin: Biosynthesis, Structure, and Biological Roles [J]. Annual Review of Plant Biology, 2016, 67(1):207.

[2]. Kosma D K, Bourdenx B, Bernard A, et al. The impact of water deficiency on leaf cuticle lipids of Arabidopsis. [J]. Plant Physiology, 2009, 151(4):1918.

[3]. Krolikowski K A, Victor J L, Wagler T N, et al. Isolation and characterization of the Arabidopsis organ fusion gene HOTHEAD[J]. The Plant Journal, 2003, 35(4): 501-511.

[4]. Kurdyukov S, Yephremov A. Genetic and biochemical evidence for involvement of HOTHEAD in the biosynthesis of long-chain a-, w-dicarboxylic fatty acids and formation of extracellular matrix [J]. Planta, 2006, 224(2):315.

[5]. Xu Y, Liu S, Liu Y, et al. HOTHEAD-Like HTH1 is Involved in Anther Cutin Biosynthesis and is Required for Pollen Fertility in Rice[J]. Plant and Cell Physiology, 2017: pcx063. 
[6]. Chang P C. Localization and possible functions of Arabidopsis HOTHEAD protein [J]. 2016.http://hdl.handle.net/10012/11033

[7]. Fang Y, Hu J, Xu J, et al. Identification and characterization of Minil, a gene regulating rice shoot development [J]. Journal of integrative plant biology, 2015, 57(2): 151-161.

[8]. Chen Q, Yu H, Wang X R, et al, An alternative cetyltrimethyl ammonium bromide-based protocol for RNA isolation from blackberry (Rubus L.)[J]. Genetics and Molecular Research, 2012, 11(2):1773-1782.

[9]. Comai L, Cartwright R A. A toxic mutator and selection alternative to the non-Mendelian RNA cache hypothesis for hothead reversion [J]. The Plant Cell, 2005, 17(11): 2856-2858. 